\title{
Antithyroid drug treatment and pregnancy outcomes among women with hyperthyroidism in pregnancy: A Norwegian population-based registry-linkage study
}

\author{
Nebghouha El Khalil ${ }^{1}$, Angela Lupattelli ${ }^{1}$ and Hedvig Nordeng ${ }^{1,2}$ \\ 1) PharmacoEpidemiology and Drug Safety Research Group, Department of Pharmacy, and PharmaTox \\ Strategic Research Initiative, Faculty of Mathematics and Natural Sciences, University of Oslo, Oslo, Norway \\ 2) Department of Child Health and Development, Norwegian Institute of Public Health, Oslo, Norway \\ Correspondence: Nebghouha El Khalil, PharmacoEpidemiology and Drug Safety Research Group, Department of Pharmacy, Faculty of \\ Mathematics and Natural Sciences, University of Oslo, PO Box 1068 Blindern, NO-0316 Oslo, Norway \\ E-mail: nebghoue@student.farmasi.uio.no Telephone: +4790416222
}

\begin{abstract}
Aims: The aim of this study was two-fold: i) to describe factors associated with antithyroid drug (ATD) treatment during gestation among women with hyperthyroidism in pregnancy, ii) to investigate the impact of ATD treatment during gestation on pregnancy outcomes.

Methods: Women with hyperthyroidism in pregnancy and ATD treatments were identified through linkage of three national registries (2008-2018): The Medical Birth Registry of Norway, the Norwegian Prescription Registry and the Norwegian Patient Registry. Pregnancies were categorized as ATD treated or untreated based on filled prescriptions indicating ATD exposure during pregnancy. ATD treatment was examined by trimester (T1, T2/T3) and by substance carbimazole (CMZ), propylthiouracil (PTU) and by both CMZ/PTU. Generalized estimating equations analysis with a robust variance estimator was used to estimate adjusted odds ratio (aOR) and adjusted standardized mean difference (aSMD) with 95\% confidence interval (CI).

Results: We identified 1699 pregnancies with hyperthyroidism during gestation. Hyperthyroidism was treated with ATD in $44.4 \%$ of the pregnancies, while $55.6 \%$ were untreated. Pregnant women treated with ATD had more often asthma compared to untreated women. Prenatal exposure to CMZ was associated with increased risk of preterm birth (aOR 1.8, 95\% CI 1.1-2.8) whereas PTU exposure in the first trimester was associated with an increased risk of cardiac malformations (aOR 9.0, 95\% CI 1.8-44.7). There was no association between ATD treatment in pregnancy and maternal preeclampsia (aOR $0.8,95 \%$ CI $0.4-1.3)$ and gestational hypertension (aOR $0.9,95 \%$ CI $0.5-1.8$ ).

Conclusion: This nationwide registry study found an association between treatment with carbimazole and increased risk of preterm birth. Exposure to propylthiouracil in the first trimester was associated with an increased risk of cardiac malformations. These findings should be interpreted in light of international findings on the risk of untreated hyperthyroidism and the potential risk of ATD treatment for the mother and child.
\end{abstract}

This is an open access article distributed under the Creative Commons Attribution Licence, which permits unrestricted use, distribution, and reproduction in any medium, provided the original work is properly cited.

\section{INTRODUCTION}

Hyperthyroidism is a condition with increased production of thyroid hormones in the thyroid gland. Common symptoms include unexplained weight loss, rapid heartbeat, unusual sweating, and swelling at the base of the neck as well as sleep, mood and appetite changes [1]. Prevalence estimates for hyperthyroidism in pregnancy range between $0.1 \%$ and $1.0 \%$ depending on whether overt or subclinical hyperthyroidism is included $[1,2]$. Graves' disease (GD) is the most common cause of hyperthyroidism in women of reproductive age, and the main type of overt hyperthyroidism during pregnancy. Another major cause of hyperthyroidism in pregnancy is gestational transient thyrotoxicosis (GTT), which occurs due to the stimulating action of human chorionic gonadotropin (HCG) on thyroid stimulating hormone (TSH) [3-5].

Untreated overt hyperthyroidism may have severe negative consequences for both the pregnant woman and her unborn child. Several international studies have shown an association between maternal hyperthyroidism and increased risk of preterm birth and low birth weight [6-9]. Maternal complications, such as pregnancy-induced hypertension and preeclampsia are also more common in pregnancies with hyperthyroidism [8-10].

In a retrospective study over a period of 28 years, it was found that women with uncontrolled hyperthyroidism during pregnancy had significantly higher rates of preterm birth ( $49 \%$ vs. $23 \%$ ) and higher odds for stillbirth (OR 8.42, 95\% CI 2.0-35.2) compared to women with well-controlled hyperthyroidism [11]. An inverse relationship between the degree of control of maternal hyperthyroidism and risk for low birth weight was reported [12]. Subclinical and GTT were not associated with adverse pregnancy outcomes $[13,14]$.

International and Norwegian national guidelines recommend pharmacological treatment of overt hyperthyroidism during pregnancy to maintain mild maternal 
hyperthyroidism while avoiding fetal hypothyroidism. For subclinical hyperthyroidism and GTT, however, pharmacological treatment is generally not recommended [15-17]. The treatment of choice for women with overt hyperthyroidism is antithyroid drugs (ATDs), which includes propylthiouracil (PTU), methimazole (MMI) and its prodrug carbimazole (CMZ). Use of ATDs during pregnancy poses specific challenges, as both MMI/CMZ and PTU have been associated with different adverse pregnancy outcomes including congenital malformations $[18,19]$ preterm birth, low birth weight and infant mortality [20]. In addition, MMI/ CMZ has been associated with a pattern of embryopathy including choanal atresia, esophageal atresia, omphalocele, omphalomesenteric duct anomalies, congenital heart disease, renal system malformations and aplasia cutis [21]. Due to safety concerns regarding possible teratogenic effects of MMI/CMZ, PTU has been the preferred ATD during the first trimester of pregnancy $[22,23]$. However, PTU has also been associated with increased risk of hepatoxicity [24,25]. Consequently, Norwegian and European guidelines recommend using PTU when planning pregnancy and during the first trimester, then switching to $\mathrm{MMI} / \mathrm{CMZ}$ thereafter in order to minimize the risk of PTU-related hepatotoxicity $[16,17]$.

In 2018, the European Medicines Agency (EMA) reviewed the evidence and published updated warnings about the potential risks of MMI/CMZ and PTU during pregnancy $[26,27]$. At the same time, they recommended to avoid switching between MMI/CMZ and PTU as risks seemed highest in pregnancies exposed to several ATDs. This was also confirmed in the recent systematic literature review and meta-analyses where the excess risk of any and major birth defects per 1000 respectively was reported to be 10.2 and 1.3 for PTU; 17.8 and 2.3 for MMI/CMZ; 32.5 and 4.1 for both MMI/CMZ and PTU; and 9.6 and 1.2 for untreated hyperthyroidism [28]. Authors highlighted that few studies have been able to account for the untreated underlying illness, and that larger studies including different treatment groups are needed to determine the impact of hyperthyroidism and ATD treatment on pregnancy outcomes [28].

To increase knowledge about treatment of hyperthyroidism in pregnancy, our aim was to i) characterize factors associated with ATD treatment during gestation among women with hyperthyroidism in pregnancy, and ii) assess the association between ATD treatment and adverse pregnancy outcomes.

\section{MATERIAL AND MethodS}

\section{Data collection}

Data were retrieved from the Medical Birth Registry of Norway (MBRN), the Norwegian Prescription Database (NorPD) and the Norwegian Patient Registry (NPR). These databases were linked based on the personal identification number assigned to everyone residing in Norway.
The Medical Birth Registry of Norway

MBRN is a nationwide birth registry with stored data on all Norwegian births after pregnancy week 12 since 1967. The MBRN contains sociodemographic information about the mother and her health before, during and after pregnancy, as well as pregnancy and birth related complications and pregnancy outcomes [29]. Previous studies have found a relative high validity of recorded information in MBRN, but inaccurate disease registration may occur [30-32].

\section{The Norwegian Prescription Database}

Established in 2004, NorPD is a nationwide registry which includes information about all redeemed prescriptions from pharmacies in Norway. NorPD classifies drugs according to the Anatomical Therapeutic Chemical (ATC) classification system. Information about drugs includes the name, dosage, package size and the date drug was redeemed [33].

\section{The Norwegian Patient Registry (NPR)}

NPR is a national administrative health registry that was established in 2008 and contains data on all activities in secondary and tertiary health care setting since 1997. Diagnostic codes in NPR follow the World Health Organization's International Classification of Diseases, version 10 (ICD-10). Several studies have reported a high quality and validity of the data in NPR $[34,35]$.

\section{Study population}

We included singleton pregnancies with a pregnancy outcome registered in MBRN from 2008 to 2018. The flow chart to obtain the final population is shown in Figure 1. To make inferences about hyperthyroidism in pregnancy, the study population was restricted to pregnancies among women with a hyperthyroidism diagnosis during gestation. Multiple pregnancies were excluded as they have additional risks of pregnancy complications and adverse outcomes.

Pregnancies among women with hyperthyroidism were categorized depending on ATD treatment status. Two mutually exclusive groups were defined:

- Pregnancies among women who were treated with ATDs during pregnancy (ATD treated pregnancies).

- Pregnancies among women with no treatment with ATDs during pregnancy (Untreated disease comparison group).

\section{Measures}

\section{Exposure to ATD}

Information about medication use was obtained from dispensed prescriptions from NorPD. Antithyroid drugs were defined as drugs having an ATC code H03B: H03BA02 for propylthiouracil (PTU) and H03BB01 for carbimazole (CMZ). In Norway, the prodrug carbimazole is the treatment choice of patients with hyperthyroidism. There is no marketed product with methimazole (MMI) in Norway.

We defined exposure to ATD at any time in gestation as any filled ATD prescription within the last menstrual 


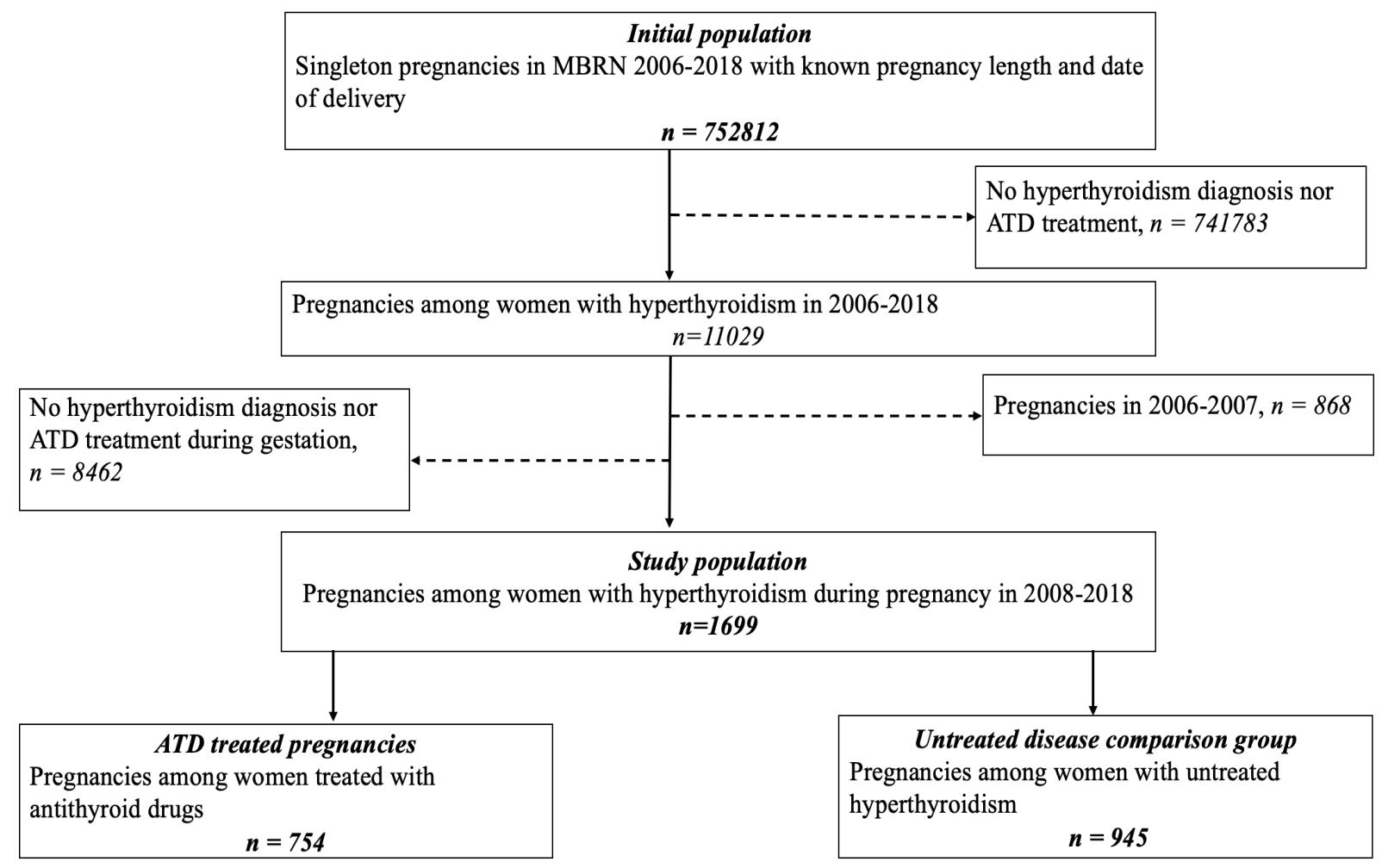

Figure 1. Flowchart of the total study sample.

period (LMP) date and delivery. Exposure to ATD during pregnancy was additionally categorized according to timing: in the first (gestational weeks 1-12), second (gestational weeks 13-26) and third trimester (from gestational week 27 to the end of pregnancy). Timing of ATD exposure was calculated based on the date of the filled prescription and the number of Defined Daily Doses (DDDs) dispensed, which overlapped with the trimester exposure windows. Pregnancies within women who filled ATD prescriptions up to the three months period before pregnancy and with dispensed DDDs overlapping with the first trimester window, were defined as ATD exposed in the first trimester.

We categorized ATD exposure into three mutually exclusive subgroups: CMZ alone, PTU alone, and both $\mathrm{CMZ}$ and PTU. The latter consisted of women with dispensed prescriptions of both of CMZ and PTU in the exposure period. Each of these was compared to the untreated disease comparison group.

\section{Maternal disease}

Diagnoses of maternal hyperthyroidism were obtained from NPR, along with the date of the diagnosis in the time period 2008 to 2018. Hyperthyroidism was defined by ICD-10 codes E05.0-E05.9. We calculated the timing of onset based on LMP data, gestational length and date of the diagnosis. Pregnancies were defined as having a diagnosis of hyperthyroidism if they had a hyperthyroidism diagnosis registered in NPR. We distinguished between women with a new-onset hyperthyroidism in pregnancy and women with a prior history, by taking into account timing of the first diagnosis of hyperthyroidism within the study period.

\section{Sociodemographic, lifestyle and health related characteristics}

MBRN covered information on sociodemographic and lifestyle factors, reproductive history, comorbidities and medication use. Sociodemographic and lifestyle factors were as follows: maternal age, marital status, parity and smoking habits. Periconceptional folic acid use and multivitamin use were classified as yes or no depending on whether the women used them before and during pregnancy. Reproductive history included previous pregnancy loss and was defined as early miscarriage and/or stillbirth. Maternal somatic comorbidities before pregnancy included asthma, chronic renal disease, diabetes and chronic hypertension. Use of other medications prior to pregnancy (three months before gestation) included analgesics (ATC N02), antianemic preparations (ATC B03) and psychoanaleptics (ATC N06). These baseline factors were considered as potential confounders.

\section{Pregnancy outcomes}

Information on maternal and infant outcomes were obtained from MBRN. Birth year was categorized into three time periods as presented in Table 1. Maternal complications included: i) any preeclampsia, by any severity or timing of onset, defined as new-onset hypertension (systolic blood pressure of $\geq 140 / 90 \mathrm{mmHg}$ ) 
Table 1. Maternal sociodemographic and medical characteristics among women with hyperthyroidism in pregnancy.

\begin{tabular}{|c|c|c|}
\hline & $\begin{array}{l}\text { ATD treated pregnancies } \\
\qquad \mathrm{n}=754\end{array}$ & $\begin{array}{c}\text { Untreated disease } \\
\text { comparison group } \\
\mathrm{n}=945\end{array}$ \\
\hline & $\mathrm{n}(\%)$ & $\mathrm{n}(\%)$ \\
\hline New-onset hyperthyroidism in pregnancy & $272(36.1)$ & $589(62.3)$ \\
\hline First hyperthyroidism diagnosis prior to pregnancy & $482(63.9)$ & $356(37.7)$ \\
\hline \multicolumn{3}{|l|}{ Year of delivery } \\
\hline $2008-2010$ & $160(21.2)$ & $235(24.9)$ \\
\hline $2011-2014$ & $279(37.0)$ & $323(34.2)$ \\
\hline $2015-2018$ & $315(41.8)$ & $387(41.0)$ \\
\hline \multicolumn{3}{|l|}{ Maternal age at delivery (years) } \\
\hline$\leq 24$ & $62(8.2)$ & $88(9.3)$ \\
\hline $25-29$ & $211(28.0)$ & $276(29.2)$ \\
\hline $30-34$ & $259(34.4)$ & $333(35.2)$ \\
\hline$\geq 35$ & $222(29.4)$ & $248(26.2)$ \\
\hline \multicolumn{3}{|l|}{ Marital status } \\
\hline Married/cohabiting & $690(91.5)$ & $884(93.5)$ \\
\hline Other & $64(8.5)$ & $61(6.5)$ \\
\hline \multicolumn{3}{|l|}{ Number of previous births } \\
\hline 0 & $260(34.5)$ & $319(33.8)$ \\
\hline 1 & $314(41.6)$ & $403(42.6)$ \\
\hline$\geq 2$ & $180(23.9)$ & $223(23.6)$ \\
\hline \multicolumn{3}{|l|}{ Smoking during pregnancy } \\
\hline Yes & $38(5.0)$ & $39(4.1)$ \\
\hline No & $609(80.8)$ & $807(85.4)$ \\
\hline Missing & $107(14.2)$ & $99(10.5)$ \\
\hline Periconceptional folic acid use & $193(25.6)$ & $279(29.5)$ \\
\hline Multivitamin intake before and during pregnancy & $119(15.8)$ & $174(18.4)$ \\
\hline \multicolumn{3}{|l|}{ Previous pregnancy loss } \\
\hline Yes & $184(24.4)$ & $251(26.6)$ \\
\hline No & $521(69.1)$ & $655(69.3)$ \\
\hline Missing & $49(6.5)$ & $39(4.1)$ \\
\hline \multicolumn{3}{|l|}{ Comorbidities } \\
\hline Asthma & $49(6.5)$ & $41(4.3)$ \\
\hline Chronic renal disease & $<5$ & $14(1.5)$ \\
\hline Diabetes & $11(1.5)$ & $17(1.8)$ \\
\hline Chronic hypertension & $4(0.5)$ & $7(0.7)$ \\
\hline \multicolumn{3}{|l|}{ Medications prior to pregnancy } \\
\hline Analgesics & $57(7.6)$ & $76(8.0)$ \\
\hline Antianemic preparations & $16(2.1)$ & $34(3.6)$ \\
\hline Psychoanaleptics & $24(3.2)$ & $37(3.9)$ \\
\hline
\end{tabular}

Bold: p-value $<0.05$ Pearson's chi square test or Fisher's Exact test if expected cell count $<5$. Variables were dichotomized into yes or no categories, unless otherwise stated.

after gestation week 20 combined with proteinuria $(\geq$ +1 dipstick on at least 2 occasions); ii) gestational hypertension, defined as hypertension occurring after 20 weeks of gestation (systolic blood pressure $\geq 140 \mathrm{~mm}$ $\mathrm{Hg}$ and/or diastolic $\geq 90 \mathrm{~mm} \mathrm{Hg}$, or an increase $>15 \mathrm{~mm}$ $\mathrm{Hg}$ from blood pressure measured before gestational week 20).

Other pregnancy outcomes included preterm birth, defined as delivery before 37 weeks of gestation, birth weight and congenital malformations. Birth weight was examined as standardized mean (i.e., z-score as continuous variable) which indicates how many standard deviations the birth weight for a child is different from the Norwegian population mean given gestational age at delivery and sex.
Congenital malformations in the offspring were grouped as "any malformation" and "cardiac malformations". Any malformation in MBRN is defined as diagnoses within the ICD-10 class Q (i.e. congenital malformations, deformations, and chromosomal abnormalities) together with ICD-10 code P83.5 (congenital hydrocele). Chromosomal abnormalities were excluded. Cardiac malformations included any diagnosis with ICD-10 codes Q20-Q26 [36]. Due to limited study power, we could not examine individual congenital malformations.

The outcome variables were all binary (yes/no), except for birth weight (continuous variable). Specific time window for ATD exposure was defined as the first trimester for analyses involving gestational hyper- 
tension, preeclampsia and congenital malformations, and anytime during pregnancy for analyses involving preterm birth and birth weight.

\section{Statistical analysis}

Pregnancy was the unit of analysis for all statistical testing. Descriptive statistics were utilized as appropriate. Pearson chi-square test was used to compare categorical data for values in each cell more than or equal to five. The Fisher's exact test was used for all other cases. Two-sample t-Test was used for the continuous variable (birth weight in grams and as z-score). The number of pregnancies was reported as $<5$ if there were less than five cases in the category of interest.

The associations between maternal factors and ATD treatment were calculated using generalized estimating equations (GEE) with a robust variance estimator. This method was used in preference to binary logistic regression analysis to take into account clustering of multiple pregnancies (309 pregnancies, 18.2\%) within the same women).

All baseline co-variates were included in the adjusted model. Results were presented as crude and adjusted odds ratios (ORs) and 95\% confidence interval (95\% CI).

\section{Analysis on the association of ATD treatment with pregnancy outcomes}

The associations between ATD treatment and maternal and pregnancy outcomes were estimated using GEE with a robust variance estimator. The association measures are presented as adjusted OR with 95\% CI for binary outcomes (e.g., preeclampsia, congenital malformations), and as adjusted standardized mean difference (aSMD), with 95\% CI for birth weight.

In the multivariable analyses, we selected a potential confounder set for pregnancy outcomes based on substance and clinical knowledge, and prior literature [28]. This confounder set included: year of delivery, age, marital status, parity, smoking during pregnancy, previous pregnancy loss, periconceptional folic acid use and multivitamin use before and during pregnancy, use of psychoanaleptics and/or analgesics prior to pregnancy and maternal comorbidities.

Because smoking and previous pregnancy loss had missing values in respectively $12.1 \%$ and $5.2 \%$ of the study population, we created a missing value category for these variables, as these were considered important confounders. To better understand the impact of missing data and confounding by smoking and reproductive history in our analyses, we performed a subanalysis where smoking and previous pregnancy loss were not included in the multivariable model. Because use of folate and multivitamins was measured before and during pregnancy, and not solely at baseline, we conducted a sensitivity analysis excluding this variable from the set of confounders.

Moreover, because pregnancies in our sample could have either a new-onset hyperthyroidism in pregnancy or a pre-existing hyperthyroidism continuing into pregnancy, we performed a sensitivity analysis further adjusting for having a first diagnosis of hyperthyroidism prior to pregnancy (yes/no).

We also calculated the estimated excess number of preterm birth and birth defect by subtracting rates of preterm birth and birth among women with ATD treated/non-treated hyperthyroidism in pregnancy from the rates of preterm birth and birth defect in the general birthing population (per 1000 births) in Norway. The general birthing population consisted of all singleton pregnancies in our linked data file used as initial population, excluding pregnancies with hyperthyroidism. The rate per 1000 births of preterm birth, any malformation and cardiac malformations was 57.5, 46.0 and 7.6, respectively.

All statistical analyses were performed with the Statistical Package for the Social Sciences (SPSS) version 27.

\section{Ethical aspects}

This study was approved by the Regional Committee for Research Ethics - South East Norway (approval number 2018/140/REK sør øst) and by the Data Protection Officer at the University of Oslo (approval number 58033).

\section{RESULTS}

\section{Population characteristics}

The study population consisted of 1699 singleton pregnancies among women with hyperthyroidism during gestation (Figure 1). Among these, 754 (44.4\%) were treated with ATD and 945 (55.6\%) were untreated (untreated disease comparison group).

Out of 1699 pregnancies, 838 (49.3\%) were among women who had a first diagnosis of hyperthyroidism before pregnancy, while $861(50.7 \%)$ pregnancies were among women with new-onset hyperthyroidism (first diagnosis was registered during pregnancy). Among 838 pregnancies with a previous hyperthyroidism diagnosis, $482(57.5 \%)$ received treatment with ATD during gestation, while 356 (42.5\%) had untreated disease.

Out of 861 pregnancies with new-onset hyperthyroidism, $272(31.6 \%)$ received ATD treatment during gestation, while 589 (68.4\%) had untreated disease.

There were no important differences in the type of diagnostic codes registered between pregnant women with a previous hyperthyroidism diagnosis and pregnant women with a new-onset disease (Supplemental table 1).

\section{Pattern of ATD use in pregnancy}

Antithyroid drug treatment was most frequent in the first trimester of pregnancy (Table 2). PTU was the most used ATD in all trimesters of pregnancy. Out of 754 ATD treated pregnancies, 251 received treatment with CMZ alone (T1: $\mathrm{n}=200), 323$ with PTU alone (T1: $\mathrm{n}=285$ ), and 180 with both of CMZ and PTU (T1: $\mathrm{n}=130$ ). A smaller proportion of pregnancies redeemed 


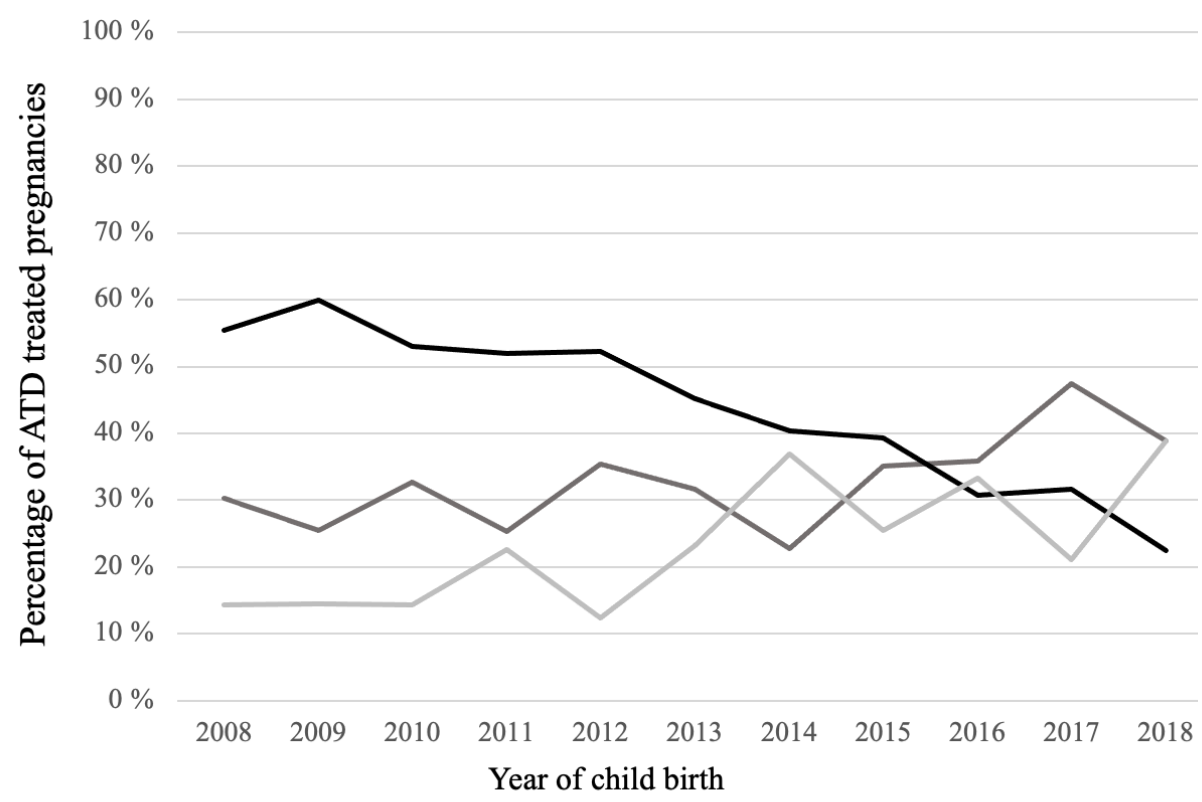

—Carbimazole - Propylthiouracil - Carbimazole and propylthiouracil

Figure 2. Time trends of use of different antithyroid drugs during pregnancy.

Table 2. Use of antithyroid drugs during gestation among women with hyperthyroidism in pregnancy in Norway between 2008 and 2018, $\mathrm{N}=1699$.

\begin{tabular}{lcccc}
\hline & Total in pregnancy & First trimester & Second trimester & Third trimester \\
& $\mathrm{n}(\%)$ & $\mathrm{n}(\%)$ & $\mathrm{n}(\%)$ & $\mathrm{n}(\%)$ \\
\hline Any antithyroid drug & $754(44.4)$ & $659(38.8)$ & $541(31.8)$ & $326(19.2)$ \\
$\%$ among ATD users & 100.0 & 100.0 & 100.0 & 100.0 \\
\hline CMZ alone & $251(14.7)$ & $208(12.2)$ & $121(7.1)$ & $103(6.1)$ \\
\% among ATD users & 33.3 & 31.6 & 22.4 & 31.6 \\
\hline PTU alone & $323(19.0)$ & $321(18.9)$ & $322(19.0)$ & $189(11.1)$ \\
\% among ATD users & 42.8 & 48.7 & 59.5 & 58.0 \\
\hline CMZ and PTU & $180(10.6)$ & $130(7.7)$ & $98(5.8)$ & $34(2.0)$ \\
\% among ATD users & 23.9 & 19.7 & 18.1 & 10.4 \\
\hline
\end{tabular}

prescriptions of both PTU and CMZ throughout pregnancy, and this was most frequent in the first trimester $(23.9 \%)$.

The median sum of DDD and interquartile range (IQR) during pregnancy was 159 (76-166) for $\mathrm{CMZ}$ and 194 DDD (134-304) for PTU, corresponding to 5- and 6-months treatment in pregnancy, respectively.

Treatment trends of hyperthyroidism during pregnancy in the period 2008-2018 are shown in Figure 2. PTU alone was the most commonly used ATD among pregnant women, but there has been a decreasing trend, with the proportion decreasing from $55.4 \%$ in 2008 to $22.4 \%$ in 2018 . The proportion of pregnancies treated with $\mathrm{CMZ}$ alone has remained stable throughout pregnancy, but with a clear increase from 2015 to 2018.

\section{Factors associated with ATD treatment in pregnancy}

Table 1 shows characteristics of the study population according to ATD treatment. The proportion of pregnancies treated with ATD was significantly higher among women with a first hyperthyroidism diagnosis prior to gestation compared to pregnant women with a new-onset hyperthyroidism during gestation.

Compared to pregnant women with untreated hyperthyroidism, women who used ATD during pregnancy had a significantly higher prevalence of asthma. No difference was detected in the year of delivery between pregnancies treated with ATD and pregnancies with untreated hyperthyroidism. Differences in factors associated with ATD treatment were generally more profound for women who used CMZ alone during pregnancy (Supplemental table 2). These women had higher parity, were more likely to smoke during pregnancy and not take multivitamins before and during pregnancy.

Differences in maternal characteristics were less prominent for pregnancies treated with both of $\mathrm{CMZ}$ and PTU (Supplemental table 2).

Table 3 presents factors related to ATD treatment during pregnancy. The odds for receiving treatment with ATD was increased in pregnant women with asthma (aOR 1.6, 95\% CI 1.1-2.5). No differences in effect estimates of the association between maternal factors and ATD treatment were found whether smoking 
Table 3. Association between maternal sociodemographic, medical characteristics and ATD treatment in pregnancy compared to pregnancies among women with untreated hyperthyroidism.

\begin{tabular}{|c|c|c|}
\hline & \multicolumn{2}{|c|}{ ATD treated pregnancies } \\
\hline & Crude OR $(95 \% \mathrm{CI})$ & Adjusted OR $(95 \% \mathrm{CI})$ \\
\hline \multicolumn{3}{|l|}{ Year of delivery } \\
\hline $2008-2010$ & $0.8(0.6,1.0)$ & $0.8(0.6,1.0)$ \\
\hline $2011-2014$ & Reference & Reference \\
\hline $2015-2018$ & $0.9(0.8,1.2)$ & $1.0(0.8,1.2)$ \\
\hline \multicolumn{3}{|l|}{ Maternal age at delivery (years) } \\
\hline$\leq 24$ & $0.9(0.6,1.3)$ & $0.8(0.6,1.2)$ \\
\hline $25-29$ & $1.0(0.8,1.3)$ & $1.0(0.8,1.3)$ \\
\hline $30-34$ & Reference & Reference \\
\hline$\geq 35$ & $1.2(0.9,1.5)$ & $1.2(0.9,1.5)$ \\
\hline \multicolumn{3}{|l|}{ Marital status } \\
\hline Married/cohabiting & Reference & Reference \\
\hline Other & $1.3(0.9,1.9)$ & $1.3(0.9,2.0)$ \\
\hline \multicolumn{3}{|l|}{ Number of previous births } \\
\hline 0 & $1.0(0.8,1.3)$ & $1.0(0.8,1.3)$ \\
\hline 1 & Reference & Reference \\
\hline$\geq 2$ & $1.0(0.8,1.3)$ & $1.0(0.8,1.3)$ \\
\hline Smoking during pregnancy & $1.3(0.8,2.0)$ & $1.2(0.8,2.0)$ \\
\hline Periconceptional folic acid use & $0.8(0.7,1.0)$ & $0.9(0.7,1.1)$ \\
\hline Multivitamin intake before and during pregnancy & $0.8(0.6,1.1)$ & $0.9(0.7,1.2)$ \\
\hline Previous pregnancy loss & $0.9(0.7,1.2)$ & $0.9(0.7,1.2)$ \\
\hline \multicolumn{3}{|l|}{ Comorbidities } \\
\hline Asthma & $1.5(1.0,2.3)$ & $1.6(1.1,2.5)$ \\
\hline Chronic renal disease & $0.3(0.1,0.9)$ & $0.3(0.1,1.1)$ \\
\hline Diabetes & $0.8(0.4,1.7)$ & $0.8(0.4,1.8)$ \\
\hline Chronic hypertension & $0.7(0.2,2.5)$ & $0.7(0.2,2.3)$ \\
\hline \multicolumn{3}{|l|}{ Medications prior to pregnancy } \\
\hline Analgesics & $0.9(0.7,1.3)$ & $0.9(0.6,1.4)$ \\
\hline Antianemic preparations & $0.6(0.3,1.1)$ & $0.6(0.3,1.0)$ \\
\hline Psychoanaleptics & $0.8(0.5,1.4)$ & $0.8(0.5,1.3)$ \\
\hline
\end{tabular}

Bold: CI not including the null. Odds ratios were calculated using the untreated disease comparison group as the reference group.

*Adjusted for all the other variables in the table. The category 'no' was used as the reference, unless otherwise stated.

and previous pregnancy loss were included or excluded as confounding factors in the multivariable analysis.

\section{Pregnancy outcomes associated with ATD treatment in pregnancy}

Tables 4 and 5 present the associations between treatment of hyperthyroidism in pregnancy and different pregnancy outcomes. Pregnancies treated with any ATD had increased risk for having infants with cardiac malformations (aOR 6.3, 95\% CI 1.4-28.7) compared to pregnancies with untreated hyperthyroidism. Children born to women treated with ATD during pregnancy weighed less than children born to pregnant women with untreated hyperthyroidism, albeit the standardized effect size was clinically negligible. No significant associations were observed between ATD treatment during pregnancy and risk of gestational hypertension and/or preeclampsia.

Children born to women who were treated with $\mathrm{CMZ}$ alone had higher risk of preterm birth (aOR 1.8, 95\% CI 1.1-2.8), and low birth weight (aSMD $-0.14,95 \% \mathrm{CI}$ $-0.27,-0.01)$ compared to the untreated disease comparison group. Compared to unexposed women without hyperthyroidism, the excess number of preterm births was 58.0 per 1000 births for $\mathrm{CMZ}$ and 11.3 per 1000 births exposed for untreated hyperthyroidism.

Children born to women treated with PTU alone in pregnancy had increased risk of cardiac malformations (aOR 9.0, 95\% CI 1.8-44.7) and low birth weight. The excess number of cardiac malformations was 13.5 per 1000 births for PTU and -5.5 per 1000 births for untreated hyperthyroidism. No differences in pregnancy outcomes were observed between pregnancies treated with both of PTU and CMZ and pregnancies with untreated hyperthyroidism.

\section{Results of sensitivity analyses}

No substantial differences in risk of pregnancy outcomes between ATD treated pregnancies and the untreated disease comparison group were found in the sensitivity analysis where smoking and previous pregnancy loss were excluded from the multivariable regression model.

Subanalysis adjusted for first time diagnosis of hyperthyroidism prior to pregnancy revealed the following results (Supplemental table 3): Treatment with $\mathrm{CMZ}$ alone during pregnancy was associated with low birth weight and preterm birth (aOR 1.9, 95\% CI 
Table 4. Pregnancy outcomes associated with ATD treatment in pregnancy.

\begin{tabular}{|c|c|c|c|c|c|}
\hline & \multirow[b]{2}{*}{$\begin{array}{c}\text { Untreated disease } \\
\text { comparison group } \\
n=945\end{array}$} & \multirow{2}{*}{$\begin{array}{c}\text { Pregnancies treated } \\
\text { with any ATD } \\
n=754 \\
n=659(\mathrm{~T} 1)\end{array}$} & \multicolumn{3}{|c|}{ Pregnancies treated with specific ATD } \\
\hline & & & $\begin{array}{c}\mathrm{CMZ} \\
\mathrm{n}=251 \\
\mathrm{n}=200(\mathrm{~T} 1)\end{array}$ & $\begin{array}{c}\text { PTU } \\
\mathrm{n}=323 \\
\mathrm{n}=285(\mathrm{~T} 1)\end{array}$ & $\begin{array}{c}\mathrm{CMZ} \text { and PTU } \\
\mathrm{n}=180 \\
\mathrm{n}=130(\mathrm{~T} 1)\end{array}$ \\
\hline & $\mathrm{n}(\%)$ & $\mathrm{n}(\%)$ & $\mathrm{n}(\%)$ & $\mathrm{n}(\%)$ & $\mathrm{n}(\%)$ \\
\hline \multicolumn{6}{|l|}{ Maternal complications } \\
\hline Gestational hypertension $^{1}$ & $22(2.33)$ & $14(2.12)$ & $6(3.00)$ & $4(15.38)$ & $<5$ \\
\hline Preeclampsia $^{1}$ & $39(4.13)$ & $20(3.03)$ & $5(2.50)$ & $7(2.46)$ & $6(4.62)$ \\
\hline \multicolumn{6}{|l|}{ Infant outcomes } \\
\hline Preterm birth (<37 weeks) & $65(6.88)$ & $64(8.49)$ & $29(11.55)$ & $21(6.50)$ & $14(7.78)$ \\
\hline \multicolumn{6}{|l|}{ Birth weight } \\
\hline Mean grams (sd) & $3465(646)$ & 3383 (612) & 3354 (597) & $3372(628)$ & $3442(603)$ \\
\hline z-score, mean (sd) & $-0.04(1.02)$ & $-0.17(0.94)$ & $-0.20(0.89)$ & $-0.20(0.94)$ & $-0.08(1.01)$ \\
\hline \multicolumn{6}{|l|}{ Malformations ${ }^{1}$} \\
\hline Any & $46(4.87)$ & $25(3.79)$ & $5(2.50)$ & $10(3.51)$ & $9(6.92)$ \\
\hline Cardiac & $<5$ & $9(1.37)$ & $0(0.00)$ & $6(2.11)$ & $<5$ \\
\hline
\end{tabular}

${ }^{1}$ Restricted to ATD treatment in the first trimester of pregnancy (T1). ATD: Antithyroid drugs, CMZ: Carbimazole, PTU: Propylthiouracil. Bold: p-value $<0.05$ Pearson's chi square test or Fisher's Exact test if expected cell count $<5$. Two-sample t-Test was used for birth weight.

Table 5. Association between pregnancy outcomes and ATD treatment during pregnancy compared to pregnancies among women with untreated hyperthyroidism.

\begin{tabular}{|c|c|c|c|c|}
\hline & \multirow{2}{*}{$\begin{array}{c}\text { Pregnancies treated } \\
\text { with any ATD } \\
n=754 \\
n=659(T 1)\end{array}$} & \multicolumn{3}{|c|}{ Pregnancies treated with specific ATD } \\
\hline & & $\begin{array}{c}\text { CMZ } \\
n=251 \\
n=200(T 1)\end{array}$ & $\begin{array}{c}\text { PTU } \\
n=323 \\
n=285(T 1)\end{array}$ & $\begin{array}{c}\mathrm{CMZ} \text { and PTU } \\
\mathrm{n}=180 \\
\mathrm{n}=130(\mathrm{~T} 1)\end{array}$ \\
\hline & $\mathrm{aOR}(95 \% \mathrm{CI})$ & $\mathrm{aOR}(95 \% \mathrm{CI})$ & $\mathrm{aOR}(95 \% \mathrm{CI})$ & $\mathrm{aOR}(95 \% \mathrm{CI})$ \\
\hline \multicolumn{5}{|l|}{ Maternal complications } \\
\hline Gestational hypertension $^{1}$ & $0.9(0.5,1.8)$ & $1.3(0.5,3.1)$ & $0.6(0.2,2.0)$ & $0.6(0.1,2.9)$ \\
\hline Preeclampsia $^{1}$ & $0.8(0.4,1.3)$ & $0.6(0.2,1.5)$ & $0.6(0.2,1.3)$ & $1.3(0.6,3.1)$ \\
\hline \multicolumn{5}{|l|}{ Infant outcomes } \\
\hline Preterm birth $(<37$ weeks $)$ & $1.3(0.9,1.9)$ & $1.8(1.1,2.8)$ & $1.0(0.6,1.6)$ & $1.2(0.6,2.2)$ \\
\hline Birth weight, aSMD $(95 \% \mathrm{CI})$ & $-0.12(-0.21,-0.03)$ & $-0.14(-0.27,-0.01)$ & $-0.14(-0.27,-0.02)$ & $-0.05(-0.21,0.11)$ \\
\hline \multicolumn{5}{|l|}{ Malformations ${ }^{1}$} \\
\hline Any & $0.7(0.4,1.2)$ & $0.5(0.2,1.1)$ & $0.6(0.3,1.2)$ & $1.4(0.7,3.0)$ \\
\hline Cardiac & $6.3(1.4,28.7)$ & NA & $9.0(1.8,44.7)$ & $2.7(0.3,29.0)$ \\
\hline
\end{tabular}

${ }^{1}$ Restricted to treatment in the first trimester of pregnancy (T1).

ATD: Antithyroid drugs, CMZ: Carbimazole, PTU: Propylthiouracil. NA: Not available (no statistics were performed, because this group did not include any cases with cardiac malformations). aOR: adjusted odds ratio, CI: confidence interval, aSMD: adjusted standardized mean difference.

Bold: CI not including the null. The reference group was the untreated disease comparison group. Gestational hypertension, preeclampsia, preterm birth and birth weight were adjusted for the standard set of confounders. Malformations were adjusted for standard set of confounders, except for use of psychoanaleptics/analgesics prior to pregnancy.

1.1-3.1). The association between exposure for PTU alone in the first trimester and cardiac malformations was attenuated and moved towards the null (aOR 6.1, 95\% CI 1.0-39.2).

\section{DISCUSSION}

Almost half of the pregnancies among women with hyperthyroidism were treated with ATD, a finding that is much higher than reported in other population-based studies, for example in Finland (27\% ATD treated in pregnancy) [9], and Taiwan (24.8\% ATD treated in pregnancy) [37]. This is probably because our study population only included pregnant women with an ICD10 diagnostic code in NPR covering the secondary heath care in Norway, and these women were more likely to have had an active disease which required treatment during gestation.

\section{Pattern of ATD use in pregnancy}

The most commonly used ATD during pregnancy was PTU, consistent with a prior Swedish population-based study [38]. This can be explained by the fact that CMZ has long been associated with a higher risk of teratogenesis [21], and PTU was therefore preferred in pregnancy. However, the proportion of pregnancies treated with PTU alone has been reduced in recent years, while the use of CMZ alone has increased. These changes in the treatment pattern of hyperthyroidism may be related to recent studies in which PTU has been associated with an increased risk of hepatoxicity [24,25].

Assuming use of 1 DDD per day, the median PTU use according to dispensed DDDs covered 6 months of pregnancy, whereas CMZ covered approximately 5 months. However, the assumption of 1 DDD per day may not be valid as ATD treatment is tailored to TSH 
and $\mathrm{T}_{4}$ serum levels. Dosage of ATDs is increased or decreased to maintain the serum free $\mathrm{T}_{4}$ at the very upper limit of the normal range [17]. Larger doses can therefore be required in cases of severe disease during pregnancy. Furthermore, the duration of use varied greatly between pregnant women. The largest dispensed DDDs of PTU in pregnancy covered 10 months, which indicates that some women used higher doses than 1 DDD per day. The difference in dispensed DDD between PTU and CMZ could therefore possibly be due to difference in hyperthyroidism severity and/or difference in accordance with prescribed and dispended DDD between the two ATDs.

The fact that PTU alone was most used throughout the pregnancy is not in line with national guidelines, which recommend switching from PTU to CMZ after the first trimester of pregnancy [17]. This gives reason to strengthen knowledge of the guidelines among clinicians.

\section{Factors associated with ATD treatment in pregnancy}

Having asthma was the only factor found to be associated with ATD treatment among women with hyperthyroidism in pregnancy. This could possibly be due to a closer follow-up in pregnancy, and more possibilities to detect hyperthyroidism, and/or by a common etiological pathway [39]

To our knowledge, there is no study that has evaluated factors associated with ATD treatment among women with hyperthyroidism in pregnancy. However, a Swedish and a Danish study that compared ATD treated pregnant women with women with no ATD use and no diagnosis of hyperthyroidism reported that pregnant women with ATD treatment were older and more often smokers compared to the population comparison group [20,38].

\section{Pregnancy outcomes associated with ATD treatment in pregnancy}

Our study results suggest that adverse pregnancy outcomes as preterm birth and low birth weight are higher among pregnant women with ATD treated hyperthyroidism, but that the risks are different according to type of ATD treatment. Increased risk of low birth weight was detected in pregnancies exposed to CMZ alone or PTU alone, while preterm birth was only detected in pregnancies exposed to $\mathrm{CMZ}$ alone.

The effect size of low birth weight, however, was very small and in our opinion, not of clinical significance. Our findings are not in line with results from an American study, which revealed that pregnant women with untreated hyperthyroidism were more likely to deliver prematurely than women with treated hyperthyroidism in pregnancy [12]. On the other hand, previous studies have shown that even ATD treated hyperthyroidism was associated with up to 2.3-fold increased risk of preterm birth [7,9].

\section{Congenital malformations}

Our results suggest that infants exposed to ATD/PTU in the first trimester are at higher risk of cardiac malforma- tions compared to infants of women with untreated hyperthyroidism. However, due to the low number of exposed cases of cardiac malformations, the effect estimate was imprecise as illustrated by the wide confidence interval. The effect estimated decreased considerably when adjusting for a first-time diagnosis of hyperthyroidism prior to pregnancy. This may be a chance finding or may point to difference in the underlying severity or ability to reach adequate ATD treatment of prior and new-onset hyperthyroidism in pregnancy. Residual confounding by disease severity may thus be present.

This finding is inconsistent with previous studies where no increased risk of cardiac malformations after PTU exposure have been reported $[38,40]$. The long duration of PTU use compared to $\mathrm{CMZ}$ as indicated by dispensed DDDs, however, points to more severe hyperthyroidism, and as such causal inference of the finding is difficult to make. Moreover, we cannot exclude that residual confounding by maternal life-style behaviors, such as alcohol use and genetics, could explain our results. Further, due to low study power, we could not conduct dose-relation analyses to evaluate whether the risk may increase with greater doses or longer exposure durations, and this limits inference of our findings. This finding warrants careful interpretation and should be seen in light of recent meta-analyses of untreated hyperthyroidism and ATD treatment [28] before any recommendation for clinical practice can be made.

The critical period for each organ system varies throughout the embryonic stage [41,42]. The exact drug exposure time is therefore of great importance for the pattern of congenital malformations. This could contribute to the lack of consistency among studies in terms of the spectrum of ATD-associated congenital malformations $[6,40,43]$.

Interestingly, we did not detect any increased risk of malformations with exposure of $\mathrm{CMZ}$ alone in the first trimester. Due to small sample size, we could not assess risk for birth defects and malformation patterns previously found in other studies $[28,43]$. We can therefore not rule out such an association.

No significant association was found between exposure to both of CMZ and PTU in the first trimester and increased risk of congenital malformations as reported in prior studies [28]. However, the results are based on only 9 first trimester exposed malformed cases, and less than 5 first trimester exposed cases with a cardiac malformation. Any association can therefore not be ruled out.

\section{Strengths and limitations}

Our study is based on national health registries with nationwide coverage covering a time period over ten years. The population size allowed for analysis on individual antithyroid drugs. Important information on maternal characteristics, lifestyle factors and maternal medical complications enabled us to control for several important confounding factors. Another important 
strength with our study is being a prospectively registered nationwide study, which eliminates recall and selection bias. The validity of information on several pregnancy outcomes (gestational age, birth weight, preeclampsia and gestational hypertension) in MBRN has shown to be very high, which is required to produce robust results $[44,45]$.

Using a separate category to indicate missing can potentially introduce bias. However, in subanalysis, no differences in results in the multivariable analyses were found whether smoking and previous pregnancy loss were included or excluded as confounding factors. This suggests that these factors do not act as important confounders in the context of this study. As a result, it is reasonable to assume that missing data did not significantly affect the results of this study.

In the sensitivity analysis excluding periconceptional folic acid use and multivitamin use from the set of confounders, the effect estimates for pregnancy outcomes did not change and remained exactly the same. This suggests that these variables were only weak confounders and not intermediates.

The most important limitation of this study is that we did not have information on the severity of hyperthyroidism or the level of control of the disease. Residual confounding by maternal disease may therefore be present. Even though we adjusted for pre-existing hyperthyroidism in one subanalysis, we could not fully distinguish between new-onset and pre-existing disease in pregnancies registered in 2008-2009, due to shorter look-back time available. Yet, because of the transient nature of hyperthyroidism, this is not considered to add substantial risk of confounding beyond that of disease severity. Moreover, a limitation of using NorPD is the fact that dispensed prescriptions do not reflect actual medication use. Exposure misclassification may occur, as we do not know whether the women actually took their medications or not. In addition, NorPD did not contain information on the prescribed doses of antithyroid drugs, so we had to assume DDD reflected prescribed daily doses, which may be differentially correct or incorrect between PTU and CMZ. Furthermore, the definition of ATD exposure assumes that the drugs has been used in the same period as they were dispensed. Since this is not always the case, the correct exposure time may differ from what is calculated in this study. This is particularly important when studying congenital malformations and first trimester exposures. A possible exposure misclassification is probably non-differential and may result in a bias towards the null. This may have underestimated our effect measures.

\section{CONCLUSION}

This nationwide registry study found that women who had asthma were more likely to receive ATD treatment during pregnancy. Prenatal exposure to $\mathrm{CMZ}$ was associated with increased risk of preterm birth. First trimester PTU exposure was associated with an increased risk of cardiac malformations, which warrants further investigation. These findings should be interpreted in light of international findings on the risk of untreated hyperthyroidism and the potential risk of ATD treatment for the mother and child.

Denne artikkelen er basert på en masteroppgave fra Universitetet i Oslo, 2021:

Nebghouha El Khalil: Tyreoideasykdom i svangerskapet En populasjonsbasert kohortstudie basert på datamateriale fra fire norske helseregistre

\section{REFERENCES}

1. Cooper DS, Laurberg P. Hyperthyroidism in pregnancy. Lancet Diabetes Endocrinol 2013;1(3):238-249.

2. Andersen SL, Olsen J, Carlé A, Laurberg P. Hyperthyroidism incidence fluctuates widely in and around pregnancy and is at variance with some other autoimmune diseases: A Danish population-based study. $J$ Clin Endocrinol Metab 2015;100(3):1164-1171.

3. Alexander MG, Jorge HM. Transient non-autoimmune hyperthyroidism of early pregnancy. $J$ Thyroid Res 2011;2011:142413-142411.

4. Andersen SL, Laurberg P. Managing hyperthyroidism in pregnancy: current perspectives. Int $J$ Womens Health 2016;8:497-504.

5. Labadzhyan A, Brent GA, Hershman JM, Leung AM. Thyrotoxicosis of pregnancy. J Clin Transl Endocrinol 2014;1(4):140-144.

6. Andersen SL, Olsen J, Wu CS, Laurberg P. Low birth weight in children born to mothers with hyperthyroidism and high birth weight in hypothyroidism, whereas preterm birth is common in both conditions: A Danish national hospital register study. Eur Thyroid J 2013;2(2):135-144.

7. Luewan S, Luewan S, Chakkabut $\mathrm{P}$, Chakkabut $\mathrm{P}$, Tongsong T, Tongsong T. Outcomes of pregnancy complicated with hyperthyroidism: a cohort study. Arch Gynecol Obstet 2011;283(2):243-247.

8. Männistö T, Mendola P, Grewal J, Xie Y, Chen Z, Laughon SK. Thyroid diseases and adverse pregnancy outcomes in a contemporary US cohort. J Clin Endocrinol Metab 2013;98(7):2725-2733.

9. Turunen S, Vääräsmäki M, Lahesmaa-Korpinen AM, Leinonen MK, Gissler M, Männistö T, et al. Maternal hyperthyroidism and pregnancy outcomes: A population-based cohort study. Clin Endocrinol 2020;93(6):721728. 
10. Medici M, Korevaar TIM, Schalekamp-Timmermans S, Gaillard R, de Rijke YB, Visser WE, et al. Maternal early-pregnancy thyroid function is associated with subsequent hypertensive disorders of pregnancy: The Generation R Study. J Clin Endocrinol Metab 2014;99(12):E2591-E2598.

11. Aggarawal N, Suri V, Singla R, Chopra S, Sikka P, Shah VN, et al. Pregnancy outcome in hyperthyroidism: A case control study. Gynecol Obstet Invest 2014;77(2):94-99.

12. Millar LK, Wing DA, Leung AS, Koonings PP, Montoro MN, Mestman JH. Low birth weight and preeclampsia in pregnancies complicated by hyperthyroidism. Obstet Gynecol 1994;84(6):946-949.

13. Casey BM, Dashe JS, Wells CE, McIntire DD, Leveno KJ, Cunningham FG. Subclinical hyperthyroidism and pregnancy outcomes. Obstet Gynecol 2006;107(2, Part 1):337-341.

14. Kinomoto-Kondo S, Kinomoto-Kondo S, Umehara N, Umehara N, Sato S, Sato S, et al. The effects of gestational transient thyrotoxicosis on the perinatal outcomes: a case-control study. Arch Gynecol Obstet 2017;295(1): 87-93.

15. Alexander EK, Pearce EN, Brent GA, Brown RS, Chen H, Dosiou C, et al. 2017 Guidelines of the American Thyroid Association for the diagnosis and management of thyroid disease during pregnancy and the postpartum. Thyroid 2017;27(3):315-389.

16. Kahaly GJ, Bartalena L, Hegedüs L, Leenhardt L, Poppe K, Pearce SH. 2018 European Thyroid Association Guideline for the management of Graves' hyperthyroidism. Eur Thyroid J 2018;7(4):167-186.

17. Norheim I, Åsvold BO, Nedrebø BG. Hypertyreose og svangerskap: Norsk Selskap For Endokrinologi; 2020 [cited 2020 30.11]. Available from:

https://www.endokrinologi.no/index.php?action=showtopic\&topic=PPArRJSW.

18. Andersen SL, Olsen J, Laurberg P. Antithyroid drug side effects in the population and in pregnancy. J Clin Endocrinol Metab 2016;101(4):1606-1614.

19. Momotani N, Yoshimura Noh J, Ishikawa N, Ito K. Effects of propylthiouracil and methimazole on fetal thyroid status in mothers with Graves' hyperthyroidism. J Clin Endocrinol Metab 1997;82(11):3633-3636.

20. Schurmann L, Hansen AV, Garne E. Pregnancy outcomes after fetal exposure to antithyroid medications or levothyroxine. Early Hum Dev 2016;101:73-77.

21. The portal for rare diseases and orphan drugs. Methimazole embryofetopathy [cited 2021 16.02]. Available from: https://www.orpha.net/consor/cgi-bin/OC_Exp.php?Lng=GB\&Expert=1923.

22. Andersen SL, Olsen J, Wu CS, Laurberg P. Severity of birth defects after propylthiouracil exposure in early pregnancy. Thyroid 2014;24(10):1533-1540.

23. De Groot L, Abalovich M, Alexander EK, Amino N, Barbour L, Cobin RH, et al. Management of thyroid dysfunction during pregnancy and postpartum: An Endocrine Society Clinical Practice Guideline. J Clin Endocrinol Metab 2012;97(8):2543-2565.

24. Akmal A, Kung J. Propylthiouracil, and methimazole, and carbimazole-related hepatotoxicity. Expert Opin Drug Saf 2014;13(10):1397-1406.

25. Wang MT, Lee WJ, Huang TY, Chu CL, Hsieh CH. Antithyroid drug-related hepatotoxicity in hyperthyroidism patients: a population-based cohort study. Br J Clin Pharmacol 2014;78(3):619-629.

26. PRAC recommendations on signals: European Medicines Agency; 2018 [cited 2021 16.02]. Available from: https://www.ema.europa.eu/en/documents/prac-recommendation/prac-recommendations-signals-adopted-2629-november-2018-prac-meeting_en.pdf.

27. PRAC recommendations on signals: European Medicines Agency, 2019 [cited 2021 16.02]. Available from: https://www.ema.europa.eu/en/documents/prac-recommendation/prac-recommendations-signals-adopted-1114-june-2019-prac-meeting_en.pdf.

28. Morales DR, Fonkwen L, Nordeng HME. Antithyroid drug use during pregnancy and the risk of birth defects in offspring: systematic review and meta-analysis of observational studies with methodological considerations. Br J Clin Pharmacol 2021; Online ahead of print, doi: 10.1111/bcp.14805.

29. Irgens LM. The Medical Birth Registry of Norway; a source for epidemiological and clinical research. Scand $J$ Rheumatol 1998;27(7):105-108.

30. Engeland A, Bjørge T, Daltveit AK, Vollset SE, Furu K. Validation of disease registration in pregnant women in the Medical Birth Registry of Norway. Acta Obstet Gynecol Scand 2009;88(10):1083-1089.

31. Skomsvoll JF, Østensen M, Baste V, Irgens LM. Validity of a rheumatic disease diagnosis in the Medical Birth Registry of Norway: Validity of a diagnosis in the MBRN. Acta Obstet Gynecol Scand 2002;81(9):831-834.

32. Vikanes $\AA$, Magnus P, Vangen S, Lomsdal S, Grjibovski AM. Hyperemesis gravidarum in the Medical Birth Registry of Norway - a validity study. BMC Pregnancy Childbirth 2012;12(1):115-115.

33. Furu K. Establishment of the nationwide Norwegian Prescription Database (NorPD) - new opportunities for research in pharmacoepidemiology in Norway. Norsk Epidemiologi 2009;18(2):129-136.

34. Øie LR, Madsbu MA, Giannadakis C, Vorhaug A, Jensberg H, Salvesen Ø, et al. Validation of intracranial hemorrhage in the Norwegian Patient Registry. Brain Behav 2018;8(2):e00900. 
35. Varmdal T, Bakken IJ, Janszky I, Wethel T, Ellekjær H, Rohweder G, et al. Comparison of the validity of stroke diagnoses in a medical quality register and an administrative health register. Scand J Public Health 2016;44(2): 143-149.

36. EUROCAT Guide 1.4: Instruction for the registration of congenital anomalies: EUROCAT Rentral registry, University of Ulster, 2013 [cited 2021 22.02]. Available from: https://eu-rd-platform.jrc.ec.europa.eu/sites/default/files/Full_Guide_1_4_version_28_DEC2018.pdf.

37. Chen $\mathrm{CH}$, Xirasagar S, Lin CC, Wang LH, Kou YR, Lin HC. Risk of adverse perinatal outcomes with antithyroid treatment during pregnancy: a nationwide population-based study. BJOG 2011;118(11):1365-1373.

38. Andersen SL, Lönn S, Vestergaard P, Törring O. Birth defects after use of antithyroid drugs in early pregnancy: a Swedish nationwide study. Eur J Endocrinol 2017;177(4):369-378.

39. Luong Kvq, Nguyen LTH. Hyperthyroidism and asthma. J Asthma 2000;37(2):125-130.

40. Seo GH, Kim TH, Chung JH. Antithyroid drugs and congenital malformations: A nationwide Korean cohort study. Ann Intern Med 2018;168(6):405.

41. Finnell RH. Teratology: General considerations and principles. J Allergy Clin Immunol 1999;103(2):S337S342.

42. Lee Gregory M, Burton VJ, Shapiro BK. Chapter 3 - Developmental Disabilities and Metabolic Disorders. Elsevier, 2015: 18-41.

43. Yoshihara A, Noh J, Yamaguchi T, Ohye H, Sato S, Sekiya K, et al. Treatment of Graves' disease with antithyroid drugs in the first trimester of pregnancy and the prevalence of congenital malformation. $J$ Clin Endocrinol Metab 2012;97(7):2396-2403.

44. Moth FN, Sebastian TR, Horn J, Rich-Edwards J, Romundstad PR, Åsvold BO. Validity of a selection of pregnancy complications in the Medical Birth Registry of Norway. Acta Obstet Gynecol Scand 2016;95(5):519527.

45. Thomsen LC, Klungsøyr K, Roten LT, Tappert C, Araya E, Baerheim G, et al. Validity of the diagnosis of preeclampsia in the Medical Birth Registry of Norway. Acta Obstet Gynecol Scand 2013;92(8):943-950.

Supplemental table 1. Diagnostic codes among pregnant women with hyperthyroidism diagnosis in pregnancy from NPR.

\begin{tabular}{lccc}
\hline & & $\begin{array}{c}\text { First diagnosis } \\
\text { in pregnancy } \\
\mathrm{n}=861^{1}\end{array}$ & $\begin{array}{c}\text { First diagnosis prior } \\
\text { to pregnancy } \\
\mathrm{n}=838^{1}\end{array}$ \\
\cline { 3 - 4 } Diagnosis & Diagnostic code & $\mathrm{n}(\%)$ & \\
\hline Thyrotoxicosis with diffuse goiter & ICD-10 E050 & $396(46.0)$ & $542(64.7)$ \\
Thyrotoxicosis, unspecified & ICD-10 E059 & $414(48.1)$ & $247(29.5)$ \\
Other thyrotoxicosis & ICD-10 E058 & $74(8.6)$ & $30(3.6)$ \\
Thyrotoxicosis with toxic single thyroid nodule & ICD-10 E051 & $<5$ & $9(1.1)$ \\
Thyrotoxicosis with toxic multinodular goiter & ICD-10 E052 & $7(0.8)$ & $10(1.2)$ \\
Thyrotoxicosis factitia & ICD-10 E054 & $5(0.6)$ & $<5$ \\
Thyrotoxic crisis & ICD-10 E055 & $0(0.0)$ & $<5$ \\
Thyrotoxicosis from ectopic thyroid tissue & ICD-10 E053 & $<5$ & $0(0.0)$ \\
\hline
\end{tabular}

\footnotetext{
${ }^{1}$ The number of pregnancies cannot be summed up in the table due to multiple diagnostic codes within the same pregnancy.
} 
Supplemental table 2. Maternal sociodemographic and medical characteristics by type of antithyroid drug used during pregnancy.

\begin{tabular}{|c|c|c|c|c|}
\hline & $\begin{array}{c}\text { Pregnancies with } \\
\text { untreated } \\
\text { hyperthyroidism } \\
n=945\end{array}$ & $\begin{array}{c}\text { Pregnancies treated } \\
\text { with CMZ } \\
n=251\end{array}$ & $\begin{array}{c}\text { Pregnancies treated } \\
\text { with PTU } \\
n=323\end{array}$ & $\begin{array}{c}\text { Pregnancies treated } \\
\text { with CMZ and PTU } \\
n=180\end{array}$ \\
\hline & $\mathrm{n}(\%)$ & $\mathrm{n}(\%)$ & $\mathrm{n}(\%)$ & $\mathrm{n}(\%)$ \\
\hline \multicolumn{5}{|l|}{ Year of delivery } \\
\hline $2008-2010$ & $235(24.9)$ & 47 (18.7) & $90(27.9)$ & $23(12.8)$ \\
\hline 2011-2014 & $323(34.2)$ & $81(32.3)$ & 133 (41.2) & $65(36.1)$ \\
\hline $2015-2018$ & $387(41.0)$ & $123(49.0)$ & $100(31.0)$ & $92(51.1)$ \\
\hline \multicolumn{5}{|l|}{ Maternal age at delivery (years) } \\
\hline$\leq 24$ & $88(9.3)$ & $23(9.2)$ & $21(6.5)$ & $18(10.0)$ \\
\hline $25-29$ & $276(29.2)$ & $69(27.5)$ & $89(27.6)$ & $53(29.4)$ \\
\hline $30-34$ & $333(35.2)$ & $83(33.1)$ & $109(33.7)$ & $67(37.2)$ \\
\hline$\geq 35$ & $248(26.2)$ & $76(30.3)$ & 104 (32.2) & $42(23.3)$ \\
\hline \multicolumn{5}{|l|}{ Marital status } \\
\hline Married/cohabiting & $884(93.5)$ & $230(91.6)$ & $297(92.0)$ & $163(90.6)$ \\
\hline Other & $61(6.5)$ & $21(8.4)$ & $26(8.0)$ & $17(9.4)$ \\
\hline \multicolumn{5}{|l|}{ Number of previous births } \\
\hline 0 & $319(26.7)$ & 87 (34.7) & $114(35.3)$ & $59(32.8)$ \\
\hline 1 & $403(42.6)$ & $86(34.3)$ & $150(46.4)$ & $78(43.3)$ \\
\hline$\geq 2$ & $223(23.6)$ & $78(31.1)$ & $59(18.3)$ & $43(23.9)$ \\
\hline \multicolumn{5}{|l|}{ Smoking during pregnancy } \\
\hline Yes & $38(5.0)$ & $19(7.6)$ & $13(4.0)$ & $6(3.3)$ \\
\hline No & $609(80.8)$ & $203(80.9)$ & $259(80.2)$ & $147(81.7)$ \\
\hline Missing & $107(14.2)$ & $29(11.6)$ & $51(15.8)$ & $27(15.0)$ \\
\hline Periconceptional folic acid use & $279(29.5)$ & $61(24.3)$ & $89(27.6)$ & $43(23.9)$ \\
\hline $\begin{array}{l}\text { Multivitamin intake before and } \\
\text { during pregnancy }\end{array}$ & $174(18.4)$ & $32(12.7)$ & $55(17.0)$ & $32(17.8)$ \\
\hline \multicolumn{5}{|l|}{ Previous pregnancy loss } \\
\hline Yes & $251(26.6)$ & $59(23.5)$ & $87(26.9)$ & $38(21.1)$ \\
\hline No & $655(69.3)$ & $176(70.1)$ & $212(65.6)$ & $133(73.9)$ \\
\hline Missing & $39(4.1)$ & $16(6.4)$ & $24(7.4)$ & $9(5.0)$ \\
\hline \multicolumn{5}{|l|}{ Comorbidities } \\
\hline Asthma & $41(4.3)$ & $18(7.2)$ & $13(4.0)$ & $18(10.0)$ \\
\hline Chronic renal disease & $14(1.5)$ & $<5$ & $0(0.0)$ & $<5$ \\
\hline Diabetes & $17(1.8)$ & $<5$ & $<5$ & $5(2.8)$ \\
\hline Chronic hypertension & $7(0.7)$ & $<5$ & $<5$ & $0(0.0)$ \\
\hline \multicolumn{5}{|l|}{ Medications prior to pregnancy } \\
\hline Analgesics & $76(8.0)$ & $20(8.0)$ & $16(5.0)$ & $21(11.7)$ \\
\hline Antianemic preparations & $34(3.6)$ & $6(2.4)$ & $<5$ & $6(3.3)$ \\
\hline Psychoanaleptics & $37(3,9)$ & $6(2.4)$ & $9(2.8)$ & $9(5.0)$ \\
\hline
\end{tabular}

Bold: $p$-value $<0.05$ Pearson's chi square test or Fisher's Exact test if expected cell count $<5$ was used to compare each treatment group with untreated hyperthyroidism. CMZ: Carbimazole, PTU: Propylthiouracil. 
Supplemental table 3. Association between pregnancy outcomes and ATD treatment during pregnancy compared to pregnancies among women with untreated hyperthyroidism adjusted for previous disease.

\begin{tabular}{|c|c|c|c|c|}
\hline & \multirow{2}{*}{$\begin{array}{c}\text { Pregnancies treated } \\
\text { with any ATD } \\
n=754 \\
n=659(\mathrm{~T} 1)\end{array}$} & \multicolumn{3}{|c|}{ Pregnancies treated with specific ATD } \\
\hline & & $\begin{array}{c}\mathrm{CMZ} \\
\mathrm{n}=251 \\
\mathrm{n}=200(\mathrm{~T} 1)\end{array}$ & $\begin{array}{c}\mathrm{PTU} \\
\mathrm{n}=323 \\
\mathrm{n}=285(\mathrm{~T} 1)\end{array}$ & $\begin{array}{c}\mathrm{CMZ} \text { and PTU } \\
\mathrm{n}=180 \\
\mathrm{n}=130(\mathrm{~T} 1)\end{array}$ \\
\hline & $\mathrm{aOR}(95 \% \mathrm{CI})$ & $\mathrm{aOR}(95 \% \mathrm{CI})$ & aOR $(95 \% \mathrm{CI})$ & aOR $(95 \% \mathrm{CI})$ \\
\hline \multicolumn{5}{|l|}{ Maternal complications } \\
\hline Gestational hypertension ${ }^{1}$ & $0.9(0.4,1.8)$ & $1.2(0.4,3.1)$ & $0.6(0.2,2.2)$ & $0.6(0.2,2.5)$ \\
\hline Preeclampsia $^{1}$ & $0.8(0.4,1.4)$ & $0.6(0.2,1.5)$ & $0.6(0.3,1.4)$ & $1.3(0.6,3.2)$ \\
\hline \multicolumn{5}{|l|}{ Infant outcomes } \\
\hline Preterm birth $(<37$ weeks $)$ & $1.4(0.9,2.1)$ & $1.9(1.1,3.1)$ & $1.0(0.6,1.7)$ & $1.2(0.6,2.3)$ \\
\hline Birth weight, aSMD $(95 \% \mathrm{CI})$ & $-0.11(-0.21,-0.02)$ & $-0.14(-0.27,-0.01)$ & $-0.13(-0.26,-0.01)$ & $-0.04(-0.20,0.12)$ \\
\hline \multicolumn{5}{|l|}{ Malformations ${ }^{1}$} \\
\hline Any & $0.6(0.4,1.0)$ & $0.4(0.2,1.1)$ & $0.6(0.3,1.2)$ & $1.3(0.6,2.8)$ \\
\hline Cardiac & $5.0(0.8,29.3)$ & NA & $6.1(1.0,39.2)$ & $2.1(0.0,126.8)$ \\
\hline
\end{tabular}

${ }^{1}$ Restricted to treatment in the first trimester of pregnancy (T1).

ATD: Antithyroid drugs, CMZ: Carbimazole, PTU: Propylthiouracil. NA: Not available (no statistics were performed, because this group did not include any cases with cardiac malformations). aOR: Adjusted odds ratio; CI: Confidence interval. aSMD: Adjusted standardized mean difference.

Bold: CI not including the null. The reference group was the untreated disease comparison group. Gestational hypertension, preeclampsia, preterm birth and birth weight were adjusted for the standard set of confounders. Malformations were adjusted for standard set of confounders, except use of psychoanaleptics/analgesics prior to pregnancy. 